\title{
THE MOBILE TERMINAL OBTAINS THE METHOD OF DETECTING THE BLOOD GLUCOSE CONCENTRATION
}

\author{
Siqi Wang ${ }^{1}$, Zhifeng Zhou ${ }^{2}$ \\ ${ }^{I}$ College of Mechanical Engineering, Shanghai University of Engineering Science, Shanghai, China \\ ${ }^{2}$ College of Mechanical Engineering, Shanghai University of Engineering Science, Shanghai, China
}

\begin{abstract}
For the elderly, the detection of blood sugar has become a part of daily life will not open less, but the current method of measuring blood sugar is more fixed and trouble. This article reviews a portable device through the test paper and mobile phone and other carriers to connect through the headset interface to blood sugar and other biological information reflected on the phone, easy to detect and record.
\end{abstract}

Keywords: Headphone interface, blood sugar, Test paper

\section{INTRODUCTION}

With the rapid growth of China's economy over the past 10 years, the rapid changes in people's lifestyles, diabetes in China has become a chronic epidemic, blood glucose concentration is an important indicator of the condition of diabetes, frequent blood glucose measurement can always grasp the disease Change, take precautions as early as possible. Mobile terminal detection of blood glucose its main role is to detect the physiological parameters of the human body, and upload parameters to the phone, involving data mining and statistical analysis and other functions do not need to complete the detection side, can be very strong performance from the phone to achieve. In this paper, the use of enzyme electrode method to detect blood glucose concentration, through the headset interface and mobile phones and other intelligent devices to communicate, the blood glucose concentration and other health information quickly displayed. It has the advantages of simple operation and convenient carrying, small test time and low single test cost.

The principle of the design instrument is to cure the glucose oxidase (GOD) on the electrode surface. When the blood drops to the electrode, The glucose will be in the role of glucose oxidase (GOD) under the action of redox reaction occurs, the resulting electrons are transferred to the electrode conductive medium, at a certain voltage (usually around 0.4-0.5) under the action of the electrode The current will change, by detecting the linear relationship between the current change and the glucose concentration to achieve the purpose of detecting blood glucose concentration. The enzyme on the blood glucose meter can be converted into a conductive material, and a current of $0.5 \mathrm{~V}$ is applied at both ends of the click. The current value is then detected. Since the current value has a linear relationship with the blood glucose concentration, calculate the formula to calculate the blood sugar concentration. The relationship between the current value and the blood glucose concentration due to many complex factors is not a simple linear relationship; the density relationship image is shown in Fig.1

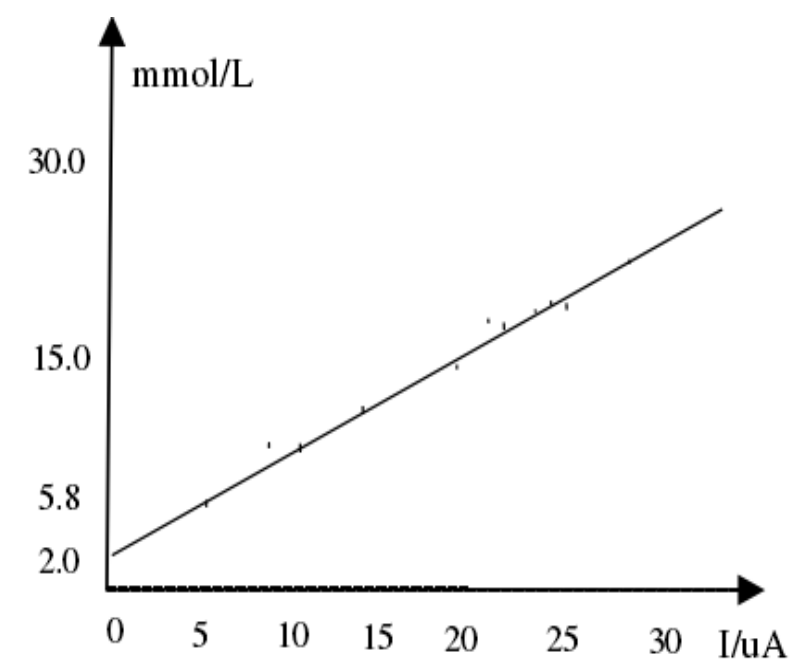

Fig.1: Relationship between current change and glucose concentration

As the mold of GOD on glucose has a high degree of heterosexuality, cannot oxidize other sugars, it can determine the true value. GOD can oxidize blood in the $\beta$ glucose to produce glucose lactone and $\mathrm{H} 2 \mathrm{O} 2$, while releasing the electrons. The specific reaction equation is as follows:

$$
\text { GLUCOSE } \stackrel{G O D}{\longrightarrow} \text { GLUCOSE LACTONE }+\mathrm{H}_{2} \mathrm{O}_{2}
$$

As the conversion current value is very small, in the measurement process we use the amplifier circuit, blood glucose in the role of glucose oxidase reaction generated by the current is extremely weak, usually a few microcaps to tens of micro amps. Dropping in the enzyme after the microcurrent generated by the electrode is very small is not easy to measure, so it will first converted into a voltage signal, and then in the amplification. Signal through the 
amplification process after the need for filtering, filtering the main purpose is to remove the interference signal, making the test more accurate. These interference signals are mainly derived from power and timing factors generated by the system noise. Get the amplified voltage signal, through the A / D converter to get digital information, easy to use the headphone jack and intelligent terminal for data communication. Amplifier circuit shown in Figure 2.

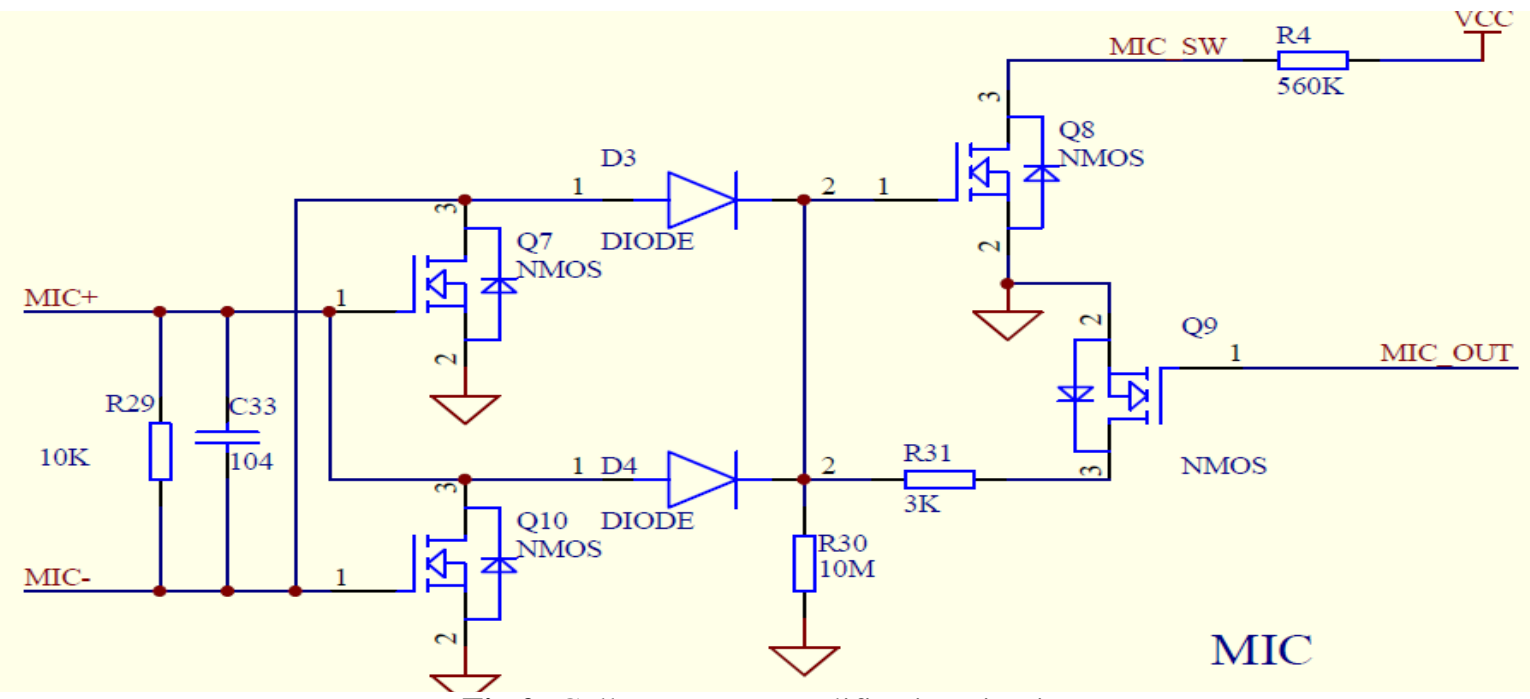

Fig.2: Collect current amplification circuit

DC power controller selection MAX603, it has a small external components, low power consumption, high efficiency and two output modes, etc., it can easily use a variety of DC power conversion circuit, not only to meet the requirements of some special systems, but also improve the performance of the instrument price.

The amplification module is designed using low-power precision op amps OP07C and LM358. OP07C is characterized by ultra-low offset, low drift, high precision, high gain, high input impedance, good circuit performance, zero offset voltage is small, and the performance is extremely stable and stable. The LM358 includes two independent, high-gain, internal frequency-compensated dual op amps that include sense amplifiers, DC gain modules, and all other single-supply power supplies that use op amps.

\section{AUDIO COMMUNICATION}

Most of the Android devices have headphone jack, under normal circumstances headphone jack is used to output audio signals to the headset, or input from the microphone to collect the audio signal, headphone pin as shown in Figure 3 .

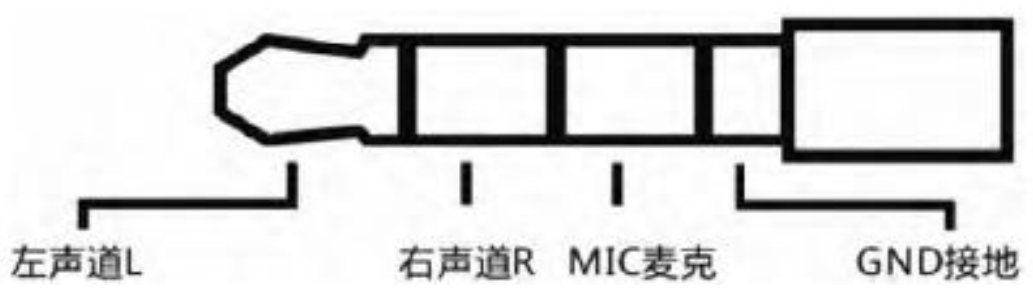

Fig 3: Headset interface definition

According to the definition of the headphone interface, you can use the left channel L (or right channel R) as the Android device output signal to the microcontroller communication line, MIC as a microcontroller output signal to the Android device communication line. The audio signal belongs to the analog signal. In order to realize the digital communication, the digital signal needs to be modulated. Headphone cable transmission is generally $1250 \mathrm{~Hz} \sim$ $9600 \mathrm{~Hz}$ between the AC audio signal 4. To carry out data transmission, the signal will need to be encoded modulation transmission, receiving demodulation decoding frequency of
$9600 \mathrm{~Hz}$ and $4800 \mathrm{~Hz}$ audio signal. The program uses the standard Manchester line coding to carry out data transmission, if the headphone interface terminal as a master device, by its generation of bit clock signal, command to select the signal and data. (1) The main control circuit module decodes the Manchester encoded data information to be transmitted by the reader / writer device into the data of the Manchester encoded data transmitted by the reader / writer device, and the main control module performs the data processing of the two-way signal through the headphone audio input / output interface and the smart 
phone. GB data, and then encoded for the differential Manchester encoded data signal, sent to the phone MIC interface or line audio input interface on the transmission line. (2) the main control circuit module from the phone headphone jack or line audio output interface such as the output $\mathrm{L}$ channel transmission line, receive the difference Manchester encoded data signal, decoded into GB data, and then encoded as standard Manchester coding data, into the MCU Built-in read and write device. Manchester code as shown in Figure 4.

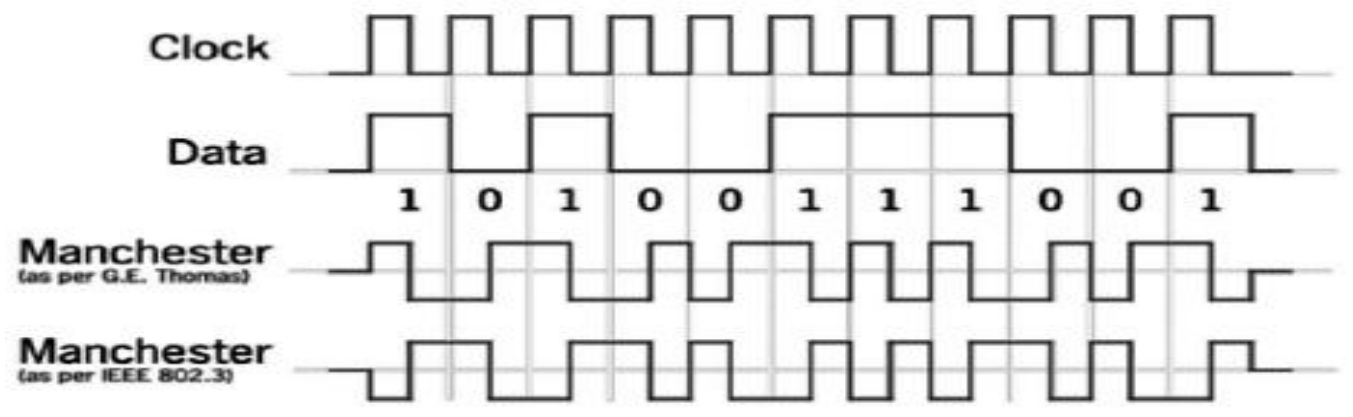

Fig.4: Manchester code

The data transmission method based on audio input / output is a serial communication method, the reference standard synchronous serial communication protocol, the use of check the Manchester coding, Manchester coding (hereinafter referred to as the code), the data transmission method is based on the audio input / Always produces an edge transition at the midpoint of each bit, and there may also be an edge transition at the beginning of each bit. The direction of the jump at each midpoint indicates the data. The resulting transition on the edge of the bit does not indicate data information. Their existence just let the signal in the correct state in order to allow every midpoint of the transition. There is a guaranteed transition to allow the signal to self-synchronize and allow the recipient to correctly match the data. The resulting data $0 \times 5 \mathrm{~A}$ is shown in Figure 5.

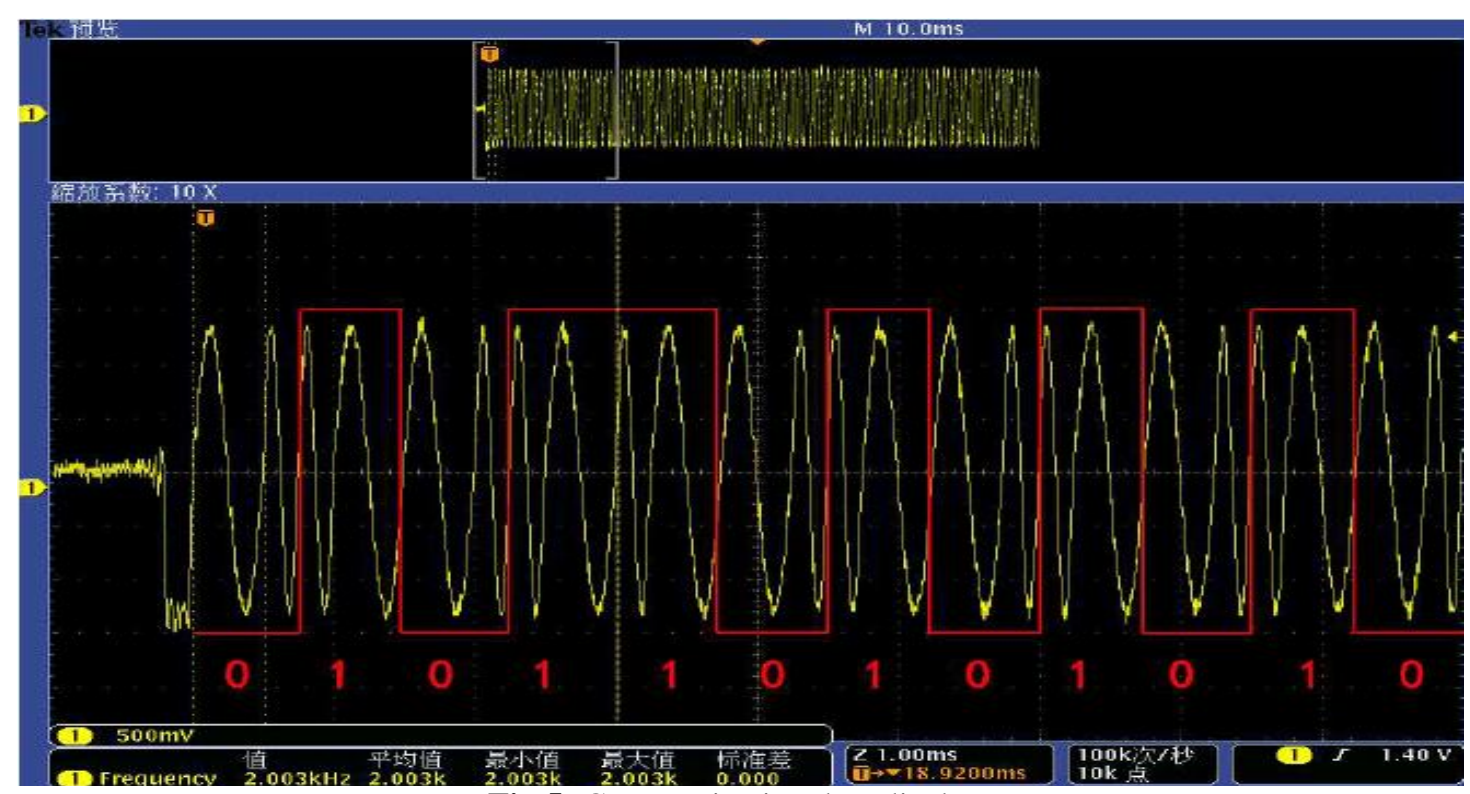

Fig.5: Communication data display

\section{DATA TRANSMISSION AND SOFTWARE DESIGN}

This design uses the ADFlash_HT66F26 microcontroller embedded firmware. On the headset peripherals, the HT66F26's built-in comparators are used to convert analog data into digital data to decode these Manchester-encoded compare data streams and continue processing them. Valid data through the program from the headset audio output interface to collect the audio signal differential Manchester encoding, the decoded data command will collect blood glucose concentration data, through the shaping circuit, and from the headset audio input MIC interface, sent to the phone for decoding The In the mobile software side, using java programming Android operating system 6-8, through the recording of the headset peripherals sent up the square wave data into PCM data. Headset peripheral software process shown in Figure 6. 


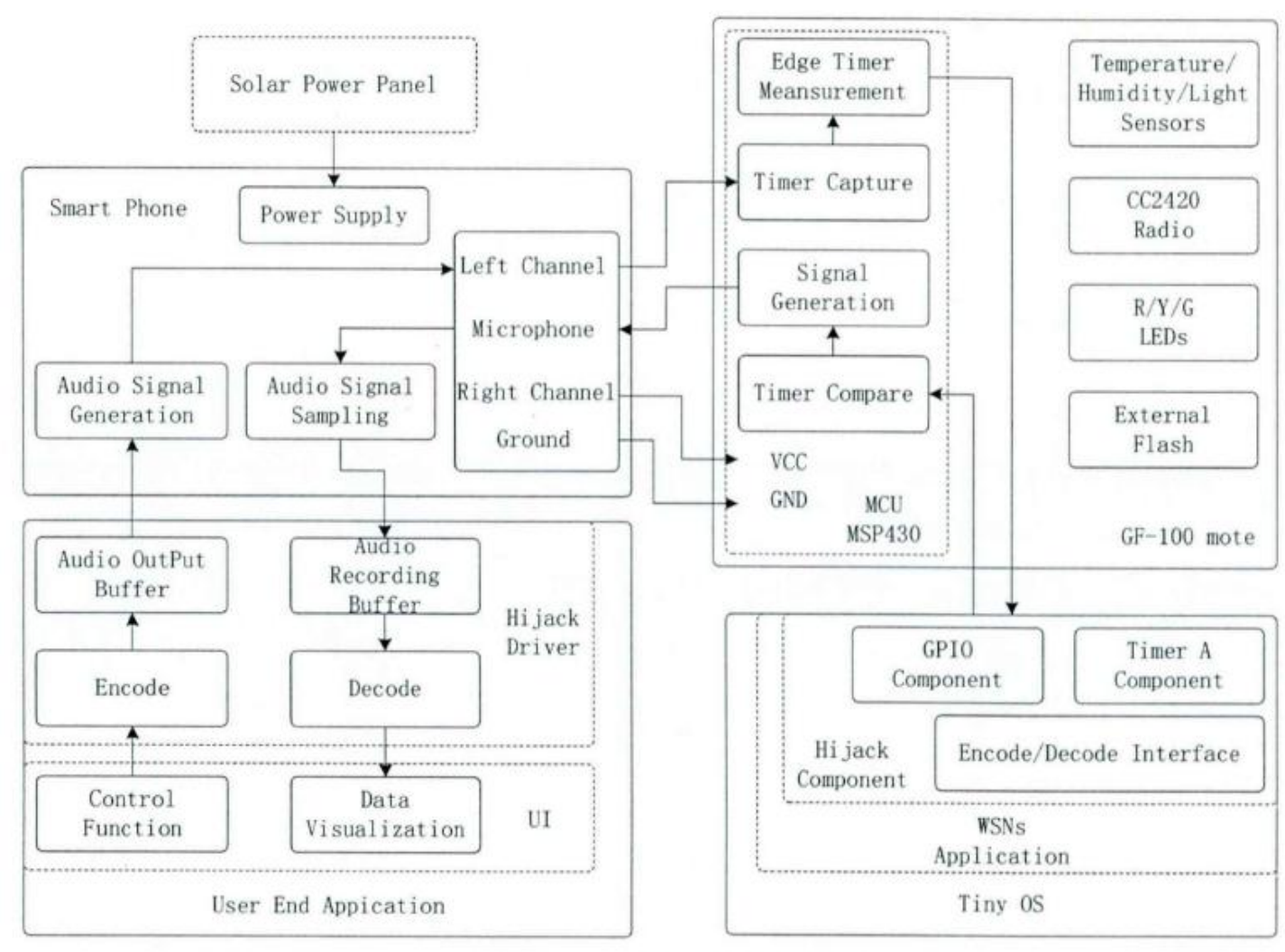

Fig.6: Block diagram of system architecture

\section{CONCLUSION}

As the limitations of mobile audio itself, the audio interface communication rate is greatly constrained, after a lot of mobile phone testing Communication rate of only about $1.3 \mathrm{Kbps}$, limited to the number of communications and communication speed requirements of the occasion. In this paper, the realization of human physiological parameters detection and mobile communication function is fully affirmed, for monitoring the terminal and mobile phone communication needs, simplifying the protocol architecture, audio communications such non-standard digital communication, from the design principles, hardware Design and optimization, modulation and demodulation, coding and decoding, transmission control and other aspects of consideration and implementation, to achieve the mobile phone and monitoring terminal standardization and standardization of communication technology.

\section{REFERENCES}

[1]. Barbara J. Philips, Jean-Xavier Meguer, Jonathan Redman, Factors determining the appearance of glucose in upper and lower respiratory tract secretions [J] . Intensive Care Medicine, 2003， 29 (12) : 2204-2210 [2]. Dan Han, Chenlei Zhang, Xiaochao Fan, at al. Understanding Android Fragmentation with Topic Analysis of Vendor-Specific Bugs[C]. Reverse Engineering (WCRE), 2012 19th Working Conference, Oct. 15-18, Kingston, Canada.
[3]. David Gay, Philip Levis, Robert von Behren, Matt Welsh, Eric Brewer and David Culler. The nes C language: A holistic approach to networked embedded systems[C]. Proceeding PLDI '03 Proceedings of the ACM SIGPLAN 2003 conference on Programming language design and implementation. New York, NY, USA: ACM New York, NY, USA, 2003:1-11.

[4]. Can Ma, Lei Wang, Zhenquan Qin, Ming Zhu,Lei Shu, Weifeng Ying, Jiaqi Xu, Improving the Throughput of Smart Phone Audio-Jack based Gateway for Sensor Networks[C]. IEEEINFOCOM 2013. Turin, Italy: IEEE Computer Society, 2013.

[5]. Aleksandar Milenkovic, Chris Otto, limil Jovanov. Wireless sensor networks for personal health monitoring: Issues and an implementation [J]. Computer Communications, 2006(29):2521-2533.

[6]. Ye-Sheng Kuo, Sonal Verma, Thomas Schick, Parball Dutta. Hijacking Power and Bandwidth from the Mobile Phone's Audio interface[C]. First Annual Symposium on Computing for Development(DEV' 10). New York, USA:ACM New York,USA,2010:24 